\title{
Multimodal imaging for clinical target volume definition in prone whole-breast irradiation: a single institution experience
}

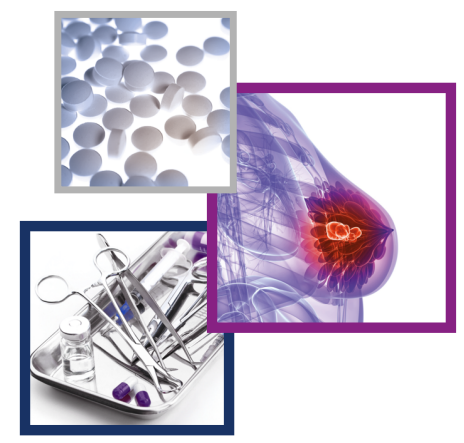
\author{
Francesca Aroldi ${ }^{4,6}$, Alberto Zaniboni ${ }^{4}$ \& Mario Bignardi ${ }^{1}$ \\ ${ }^{1}$ Radiation Oncology Unit, Fondazione Poliambulanza, Via Bissolati 57, 25124 Brescia, Italy \\ ${ }^{2}$ Radiology Department, Fondazione Poliambulanza, Via Bissolati 57, 25124 Brescia, Italy \\ ${ }^{3}$ Surgical Department, Fondazione Poliambulanza, Via Bissolati 57, 25124 Brescia, Italy \\ ${ }^{4}$ Oncology Department, Fondazione Poliambulanza, Via Bissolati 57, 25124 Brescia, Italy \\ ${ }^{5}$ Breast Unit, Fondazione Poliambulanza, Via Bissolati 57, 25124 Brescia, Italy \\ ${ }^{6}$ Università Cattolica del Sacro Cuore, Roma, Italy \\ * Author for correspondence: alessandra.huscher@poliambulanza.it
}

Alessandra Huscher*,1,5, Dina Santus 2,5, Alberto Soregaroli 2,5 , Stefano Mutti ${ }^{3,5}$, Gabriele Levrini ${ }^{2,5}$, Laura Donadoni ${ }^{1,5}$, Paolo Cascioli ${ }^{3,5}$, Francesca Guerini ${ }^{3,5}$, Tiziana Prochilo ${ }^{4,5}$,

\section{Summary points}

- No clinical target volume (CTV) contouring rules for prone breast irradiation are currently available.

- CTV contouring rules have demonstrated improved accuracy and homogeneity of radiation therapy target volumes.

- MRI provides greater soft-tissue discrimination than computed tomography (CT).

- Breast glandular tissue distribution was analyzed in a sample of diagnostic breast MRI, and the volume encompassing all breast glandular tissues was delineated (MRI CTV).

- Anatomical reference structures for MRI CTV were identified.

- Simulation MRI and CT were fused through dedicated workflow and MRI CTV deformed and transferred on CT slices.

- Anatomical reference structures for transferred CTV were identified.

- Reference structures were superimposable for MRI or CT images.

- Anatomical references for prone breast CTV were the following:

- Anteriorly: $3 \mathrm{~mm}$ from the skin.

- Posteriorly: the anterior surface of the pectoralis major muscle.

- Superiorly: the plane passing through the caudal edge of the clavicular head.

- Inferiorly: the inframammary fold.

- Laterally: a plane tangential to the external margin of the pectoralis muscles and perpendicular to the skin.

- Medially: the anterior thoracic arteries or, if not visible, the homolateral sternal margin.

- Prone breast irradiation is a nonstandard position, suitable only for selected cases. Further analysis on a greater number of samples will help in defining the accuracy of our definition.

Aim: Theaim was identification of reference structures for breast clinical target volume (CTV) in prone position, throughout image fusion process. Materials \& methods: We analyzed breast glandular tissue distribution in 20 diagnostic MRIs, referring to structures reported in ESTRO guidelines for supine irradiation. The volume containing breast glandular tissue in all cases was defined as MRI prone CTV (MRIpCTV). Then in ten subsequent patients planned for prone irradiation, MRI and computed tomography (CT) simulation was acquired. MRIPCTV was defined followed by our findings and transferred to CT for definitive delineation. Results: MRIpCTV was defined by the caudal edge of clavicular head, $3 \mathrm{~mm}$ above inframammary fold, by the medial thoracic artery, by a plane passing through the lateral surface of pectoralis muscles, by the anterior surface of pectoralis muscles and $3 \mathrm{~mm}$ from the skin. Deformed CTV was consistent with anatomy on CT; the limits chosen for MRIpCTV fit adequately also for CT. Conclusion: Prone irradiation is an alternative set up for selected cases, so the sample is very small. However, our suggestions could be of aid in defining prone CTV. The good consistency between MRI and CT seems to confirm that MRI may be unnecessary in routine practice. 
First draft submitted: 24 May 2017; Accepted for publication: 17 October 2017; Published online: 2 February 2018

Keywords: breast glandular tissue $\bullet$ breast radiotherapy $\bullet$ CTV definition $\bullet$ image fusion $\bullet$ prone $\bullet$ simulation MRI

Clinical target volume (CTV) definition is fundamental in radiation therapy, for both local control and organ at risk (OAR) irradiation. In whole-breast irradiation CTV must include all breast glandular tissue (BGT) remaining after conservative surgery and the surgical bed. Because treatment planning systems for dose calculation are based on tissue density expressed through the Hounsfield parameter, CTV contouring is carried out on computed tomography (CT) slices. BGT is a soft-tissue; therefore, its identification and differentiation from other soft tissues on CT slices has significant limits. In fact, substantial interobserver variability in target identification has been reported $[1,2]$.

In order to improve the homogeneity of CTV, scientific societies proposed contouring guidelines and reference anatomical structures [3]. Routine application of delineation guidelines resulted, in different papers, in a significant homogeneity of CTV contouring among operators [1-3]. This process reduced geographical omissions, assuring good quality treatments and allowed an accurate comparison of clinical results between different centers. Unfortunately, no contouring guidelines are available for nonstandard set-ups.

Prone breast irradiation is a recent technique developed to reduce normal tissue irradiation and improve dose distribution within the target volumes and mainly applied in large and/or pendulous breasts. In large breasts, CTV definition is particularly critical, and reducing OAR irradiation is mandatory. In a prone set-up, reference structures routinely in use for CTV contouring may be misleading owing to anatomical differences and may lead to unnecessary normal tissue irradiation. Few papers have reported indications of target volume definition in a prone set-up, most of them short and referring to skin wires [4-11]. Finally, prone radiotherapy requires intensity-modulated treatments for optimal dose distribution. Considering the high conformation of the isodoses, again, target volume definition is crucial $[6,10]$.

CT-MRI fusion for treatment planning is a standard procedure for many pathologies, such as prostate, brain, rectal or gynecological cancers, and its role in routine practice is well established. Breast simulation MRI in a supine set-up clearly improved visualization of BGT and reduced interobserver variability, but when compared with volumes obtained throughout guideline application, no need for such a resource-consuming procedure could be demonstrated $[12,13]$. In a prone set-up, in the absence of guidelines, MRI may reveal some relevant details, helping clinicians to define a correct CTV.

Therefore, we decided to extend MRI simulation, already in the clinical routine for other cancer sites in our institution, to breast cancer patients planned for prone irradiation. As a routine institutional practice, patients were candidates for prone irradiation only if a supine set-up determined excessive OAR irradiation. In practice, in selected patients with large and pendulous breasts, we opted for prone treatment. All patients involved gave their consent for both diagnostic procedures and anonymized use of images.

In order to gain a more precise idea of the spatial distribution of BGT in the prone position, we first analyzed diagnostic MRI images of 20 patients with intact breasts and delineated a volume including BGT in all cases. This was defined as 'MRI prone CTV' (MRIpCTV), and its 'reference boundaries' were derived from surrounding anatomical structures.

We subsequently used multimodal imaging co-registration on ten patients planned for whole-breast irradiation in the prone position, applying the derived contouring reference structures on simulation MRI, which was deformed and transferred to CT simulation slices, creating a deformed prone CTV (dCTCTV), which was finally corrected by a dedicated clinician, creating the CT prone CTV (CTpCTV). In the end, we also identified delineation references for CTpCTV.

\section{Materials \& methods}

This work consisted of two phases. In the first phase, we analyzed BGT distribution on 20 diagnostic breast MRIs without final evidence of disease. Therefore, we looked for intact breasts. Prone irradiation is mainly proposed for either large or pendulous breasts; therefore, patients with breast volumes smaller than $350 \mathrm{cc}$ were excluded. In all cases MRI imaging was acquired with a standard bore Scanner $\left(1.5 \mathrm{~T}\right.$ Siemens ${ }^{\odot}$, Siemens, Erlangen, Germany), with T1 and T2 sequences, with and without water and fat suppression. Patients were placed in the prone position, 
Table 1. Characteristics of patients treated with prone breast irradiation.

\begin{tabular}{|c|c|c|c|c|c|c|}
\hline Patient & Age & Bra cup size & pT & $\mathrm{pN}$ & $\mathrm{CHT}$ & HT \\
\hline 1 & 54 & 5 & $1 c$ & 0 & No & Yes \\
\hline 2 & 52 & 4 & is & 0 & No & No \\
\hline 3 & 53 & 4 & is & 0 & No & No \\
\hline 4 & 51 & 4 & is & 0 & No & No \\
\hline 5 & 52 & 4 & is & 0 & No & No \\
\hline 6 & 66 & 7 & $1 c$ & 0 & No & Yes \\
\hline 7 & 46 & 4 & $1 c$ & 0 & Yes & No \\
\hline 8 & 55 & 4 & $1 c$ & 0 & No & Yes \\
\hline 9 & 52 & 4 & $1 c$ & 0 & No & Yes \\
\hline 10 & 57 & 7 & $1 b$ & $1 a$ & No & Yes \\
\hline
\end{tabular}

with both arms anteriorly abducted, on an 8-channel device. Intravenous contrast injection was used in all cases (16 ml Multihance Bracco $^{\odot}$, Bracco, Milan, Italy).

MRIpCTV was defined as the volume including all evaluable BGT (axillary tail when visible), in all patients. A dedicated radiologist validated contoured volumes with the aim of minimizing target omissions. The anterior margin of CTV in the periareolar region was assumed to be $3 \mathrm{~mm}$ from the surface, due to the difficulty of separating glandular tissue from terminal ductal structures. Reference structures were also contoured on MRI and subsequently used for definition of boundaries as follows:

- Clavicular heads;

- Sternum;

- Anterior thoracic artery;

- Medial thoracic artery;

- External skin surface;

- Major and minor pectoralis muscles.

The MIM Vista ${ }^{\odot}$ software program (version 6.6 MIM Software Inc., OH, USA) was used for volume contouring and image fusion and deformation. We analyzed the spatial distribution of BGT with respect to the above-described anatomical structures.

In the second phase, we acquired postoperative MRI images for co-registration with CT for treatment planning in ten patients planned for prone breast irradiation after conservative surgery. All received whole-breast irradiation without nodal areas inclusion. Cup size was 4-7 (European size). Four patients were treated for ductal carcinoma in situ and six for stage I or II invasive cancers. Only one patient received chemotherapy as systemic adjuvant treatment and five patients received hormones. Table 1 reports patients' characteristics.

For treatment planning CT, patients were set on a Civco New Horizon Prone Breast Board ${ }^{\odot}$ (Civco, Kalona, IA, USA) with both arms abducted; with a Philips Big Bore $\mathrm{CT}^{\odot}$ (Philips Medica Systems, OH, USA), 3 mm thickness and $3 \mathrm{~mm}$ scans were acquired while the patient breathed freely using the whole lungs. MRI was acquired following the above-described protocol without contrast, as only BGT visualization was necessary.

MRIpCTV was defined on MRI images according to the indications derived from the first phase. MRI-CT co-registration with image-deformable fusion with a specifically dedicated workflow was used to transfer MRI CTV on CT in order to create the dCTCTV for treatment planning. The dedicated workflow consisted first of a rigid fusion based on bone structure; subsequently, after locking superimposable regions, the software deformed the MRI CTV contour to create the corresponding dCTCTV. A dedicated clinician then checked the result of image fusion and defined the final CTpCTV.

We reported mean value for all volumes of interest, with range distribution; The Student's $t$-test was used to compare the data distribution. 


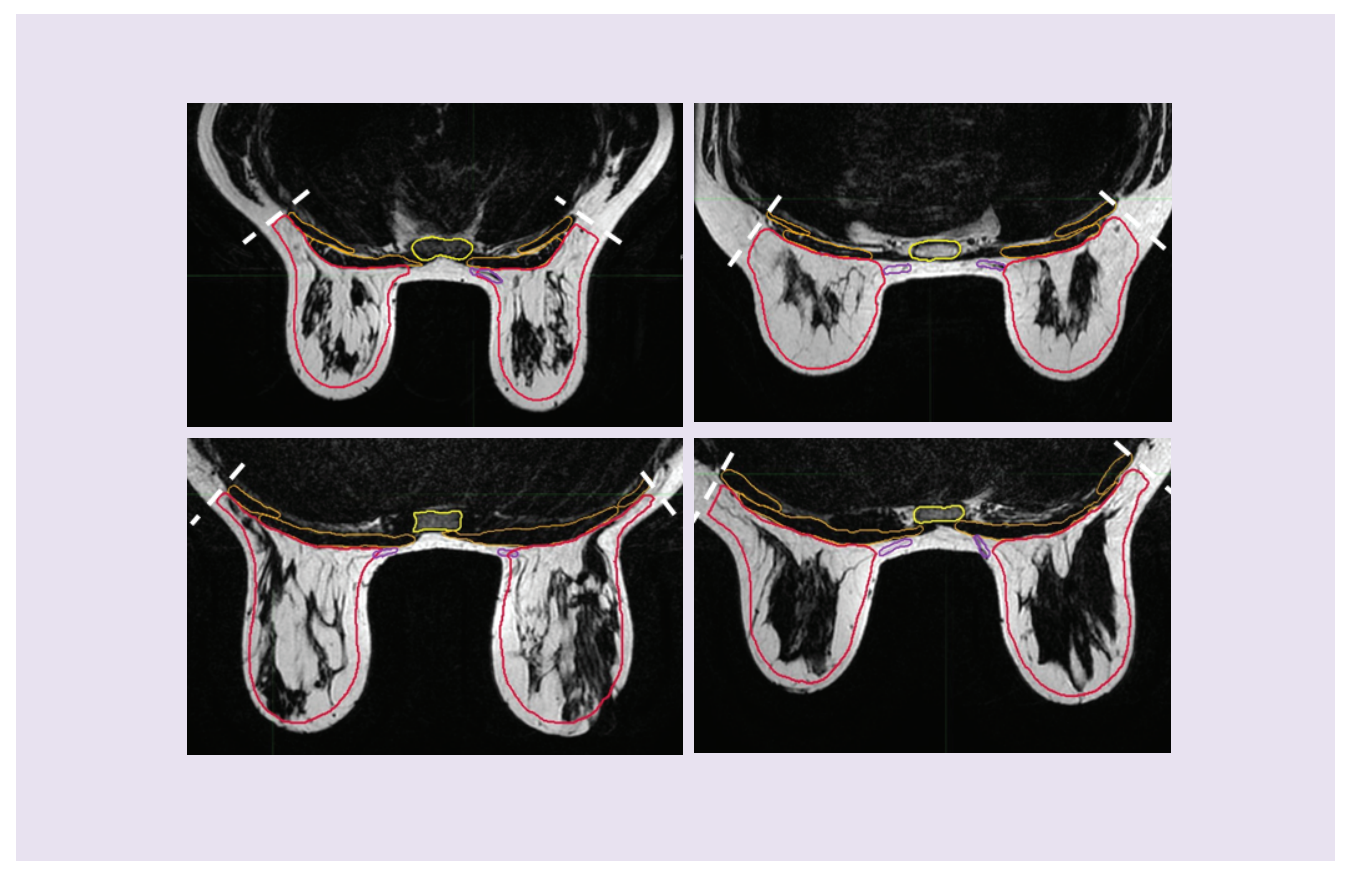

Figure 1. Examples of breast glandular tissue for intermingled (on the left) and centralized (on the right) distributions. MRI prone clinical target volume, the sternum, the pectoralis muscles and the anterior thoracic arteries are contoured. The dotted line passes through the external surface of the pectoralis, perpendicular to the skin.

\section{Results}

First phase-MRIpCTV definition

The sample analyzed included 20 patients with intact breasts and a median age of 48 years; the low mean age was probably the result of a wider application of MRI in younger patients. As described in previous literature, we could identify two different distributions of BGT within the organ; in 33\% of patients, BGT was centrally located with fat tissue mainly disposed peripherally, the so-called 'centralized' distribution. In most patients, however, BGT was uniformly distributed within fat tissue, assuming an 'intermingled' distribution [14]. Figure 1 shows examples of BGT distribution.

The two groups (centralized and intermingled) did not differ significantly in age distribution. From the analysis of BGT distribution, we concluded that no BGT was present in the first $3 \mathrm{~mm}$ from the surface (major retro-areolar ducts were, as usual, not considered); $80 \%$ of patients had no evidence of BGT in the first 5 mm below the skin surface as reported in previous literature [13]. In the cranial direction no evidence of BGT was found above a plane passing through the inferior limit of the clavicle heads; this limit frequently corresponded to the Cooper's ligament, which is not always identifiable [13]. Caudally, BGT ended 3-5 mm above the inframammary fold; we did not find BGT medially to the anterior thoracic arteries, confirming already published results. When the anterior thoracic arteries were not clearly detectable, BGT was found at least $5 \mathrm{~mm}$ from the lateral sternal margin. Posteriorly, BGT irregularly joined the anterior surface of the pectoralis muscle, while laterally we identified a plane parallel to the external margin of the pectoralis muscles and perpendicular to the skin surface as a viable limit of BGT extension. Such a plane also included the axillary tail when present.

Therefore, considering all uncertainties, we proposed the following anatomical references of MRI CTV, as reported in Table 2.

- Anteriorly: $3 \mathrm{~mm}$ from the skin.

- Posteriorly: the anterior surface of the major pectoralis muscle.

- Superiorly: the plane passing through the caudal edge of the clavicular head.

- Inferiorly: the inframammary fold.

- Laterally: a plane tangential to the external margin of the pectoralis muscles and perpendicular to the skin.

- Medially: the anterior thoracic arteries or, if not visible, the omolateral sternal margin. 


\begin{tabular}{|l|l|}
\hline Table 2. Anatomical references for prone clinical target volume definition. \\
\hline Borders & Reference structure \\
\hline Anterior & $3 \mathrm{~mm}$ from the skin surface \\
\hline Posterior & Anterior surface of the pectoralis major muscle/chest wall \\
\hline Superior & Plane passing through the caudal edge of the clavicular head \\
\hline Inferior & Inframammary fold \\
\hline Lateral & Plane tangential to the external margin of the pectoralis muscles and perpendicular to the skin \\
\hline Medial & The anterior thoracic artery or, if not visible, the omolateral sternal margin \\
\hline
\end{tabular}

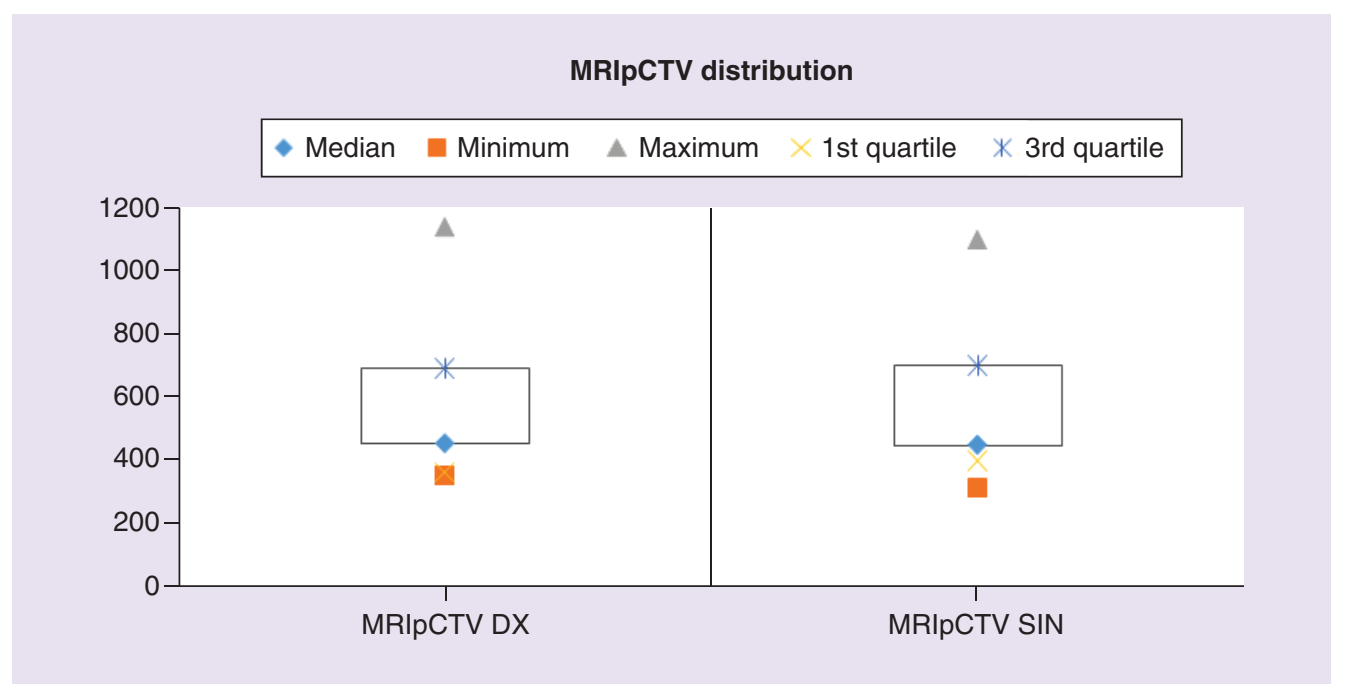

Figure 2. MRI prone clinical target volume distribution for intact breasts: right and left breast volumes are clearly consistent.

MRIpCTV: MRI prone clinical target volume.

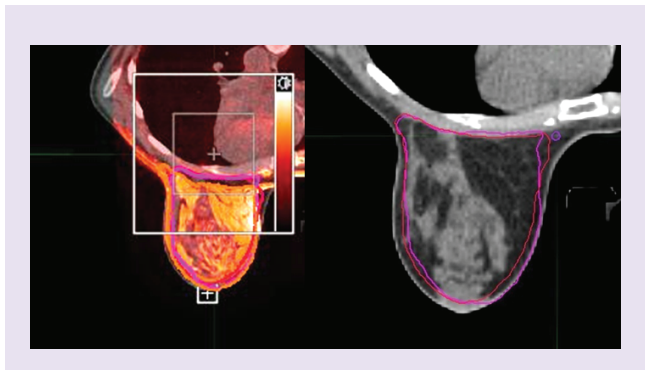

Figure 3. Image fusion process and computed tomography prone clinical target volume definition. Deformed prone clinical target volume is shown in pink and prone clinical target volume is in red. The anterior thoracic artery is shown in violet.

Mean and median right MRIpCTV volumes were, respectively, 451 and $549 \mathrm{cc}$ (range: 356-1136 cc).

Similarly, mean and median left MRIpCTV volumes were 441 and $562 \mathrm{cc}$ (range: 356-1095 cc). Clearly, right and left breast volumes were consistent. Figure 1 shows MRIpCTV contouring, while Figure 2 shows the MRIpCTV distribution for intact left and right breasts.

\section{Second phase co-registration \& CTPCTV definition}

In a preliminary comparison of CT and MRI images, we clearly recognized that the different patient set-up, particularly for contralateral breasts, determined a rotation of the trunk and subsequently a slightly different spatial distribution of BGT. In all cases, however, the image fusion process was adequately completed. We locked three-to-five areas. Figure 3 reports an example of the fusion process.

MRIpCTV, dCTCTV and CTpCTV volumes did not differ significantly. Mean MRIpCTV in this sample was 699 cc (range: 353-1207 cc), mean dCTCTV was $696 \mathrm{cc}$ (range: 360-1203 cc), and mean CTpCTV was $722 \mathrm{cc}$ 


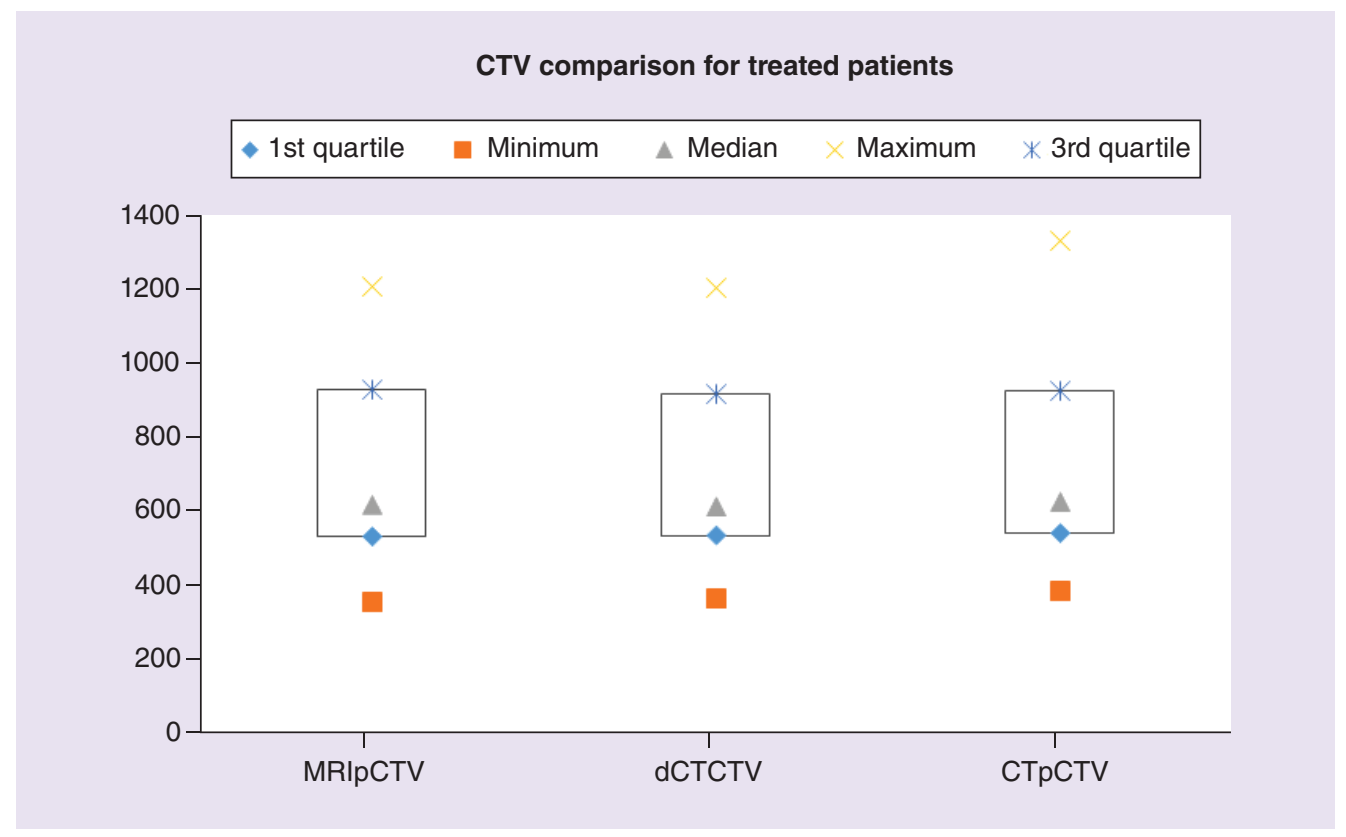

Figure 4. Box plot for clinical target volume distribution in treated patients.

CTPCTV: CT prone clinical target volume; dCTCTV: Deformed prone computed tomography clinical target volume; MRIpCTV: MRI prone clinical target volume.

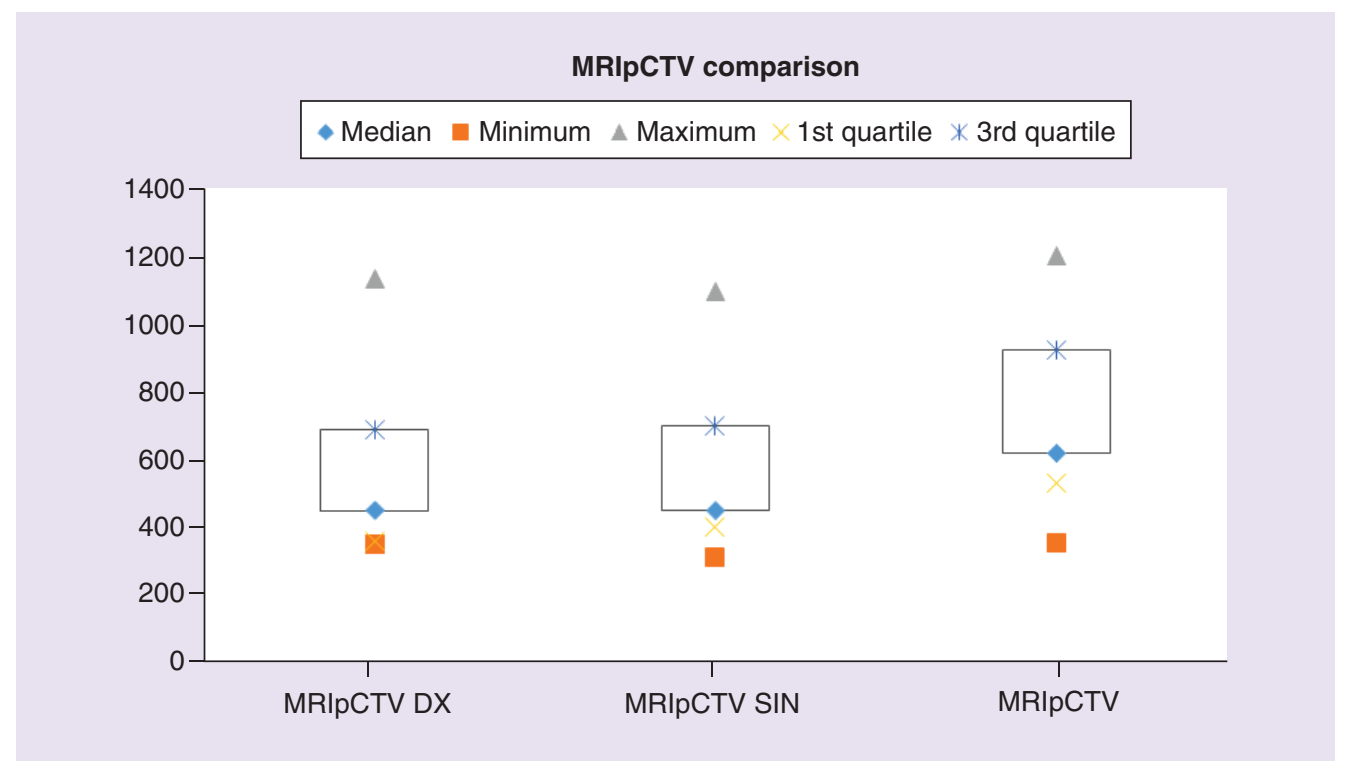

Figure 5. MRI clinical target volume distribution for intact and treated breasts (MRI prone clinical target volume). Patients subjected to prone irradiation showed higher clinical target volumes than those with intact breasts. MRIpCTV: MRI prone clinical target volume

(range: $384-1330 \mathrm{cc}$ ). The distribution of CTVs is summarized in Figure 4. The final CTpCTV, corrected by a dedicated physician, was always larger; the determinant of increasing volume seemed to be the anterior correction with a margin $3 \mathrm{~mm}$ from the skin surface. Figure 4 shows all CTV distributions. Compared with the first phase sample, MRIpCTV values were higher, as shown in Figure 5. This was due to the selection of patients with larger breasts for treatment in the prone position.

Anatomical analysis showed that although patient set-up was not exactly superimposable, the same references proposed for MRI were also fitting for CT images. The medial thoracic arteries were particularly helpful for defining 
the medial limit of CTpCTV, as already demonstrated for supine irradiation. Where the lateral limit was concerned, we found that a plane passing tangentially to the external surface of the pectoral muscles and perpendicular to the skin allowed inclusion of all BGT (axillary tail included) without the need for excessive posterior extension of CTV. Lateral thoracic arteries were, in fact, not substantial for CTpCTV delineation, probably because of significant displacement of BGT in the prone position. Pectoral fascia and skin were adequate references for the posterior and anterior margins of CTV.

\section{Discussion}

Prone irradiation is a fairly diffuse technique for breast irradiation, allowing better OAR sparing and dosimetric homogeneity in selected cases [4-11]. OAR irradiation also depends on the spatial distribution of target volumes [611]; these findings have been widely analyzed and demonstrated for different tumors and are particularly critical in modulated-intensity techniques. MRI demonstrated clear advantages in the identification of BGT and for image acquisition in the supine position [12,13]. Although of some value, due to its complexity, the related costs and limited availability, MRI cannot be proposed as a standard practice in supine breast treatment planning, as previously reported [12].

In prone radiation therapy, due to the lack of clear data on reference structures and contouring rules, MRI may play a more significant role. MRI visualization of BGT is easier; also, in sequences without contrast the diagnostic set-up position is very similar to the treatment one. Therefore, we looked at the spatial distribution of BGT on diagnostic MRI and tried to find reference structures for defining MRIpCTV for whole-breast irradiation and subsequently transfer it, through an advanced image fusion and deformation process, creating a dCTCTV on treatment CT images to achieve a definitive CTpCTV.

The first part of this work included the analysis of spatial distribution of BGT with respect to surrounding vessels and bone structures, as already shown for a supine set-up in available international guidelines.

Although the anatomy is different, we choose the same structures as in the ESTRO guidelines for supine irradiation with the intent of maintaining a certain homogeneity with already published data; the selected structures (sternum, pectoralis muscles and thoracic arteries), however, composed a good 'spatial box' for prone set-up. In fact, DiPasquale et al. have already reported similar data in their analysis of automatic segmentation for prone breasts [15]. BGT was easily identified in our sample, with distribution patterns corresponding to published data, confirming the superiority of MRI over CT in soft-tissue discrimination.

MRIpCTV volumes in the diagnostic sample were significantly lower than those of treated patients, but this was directly correlated with patient selection. As already stated, in our institution prone whole-breast irradiation is reserved for large or pendulous breasts.

We observed that the prone set-up determined, as it was already known, a reduction of craniocaudal extension of the breasts, transferable to the potential radiation field with reduction of normal tissue irradiation, in particular, lung dosimetry. Although large breasts were included, no BGT was found above the sternal notch. The medial limit of BGT in the prone position never exceeded the sternal margin and was always confined to the medial thoracic arteries, as already reported in the ESTRO guidelines for supine breast irradiation; this is not always the case in a supine set-up, and it may be helpful in reducing contralateral breast dose. A plane passing through the external surface of the pectoralis and perpendicular to skin was our lateral margin. In addition, this finding is crucial for dorsal CTV extension and OAR dosimetry. This was determined while we looked for a reproducible limit in the selected cases.

After the image fusion process applied in the second phase, MRI CTV transferred to CT appeared consistent with CT anatomical findings with clear and significant concordance. MRI-deformed CTV and subsequent dCTCTV and CTpCTV were always confined within the same anatomical boundaries, easily identifiable on a CT slice.

Posterolateral definition of CTV appeared to be of particular interest; compared with the sole definition reported in the literature [6], including all soft tissues anterior to the dorsal muscle, our margin allows a significant reduction of lateral extension of CTV and consequently of organs at risk of irradiation. Our CTV shaping, in fact, could determine smaller lung and heart (for left-sided targets) inclusion. The omission of tissues located posteriorly and laterally to the above-described plan, in a region where no evidence of BGT was found on MRI, resulted, in fact, in a less complex volume profile, facilitating normal tissue dosimetry.

A great weakness of our work is the sample size, which is clearly very small. However, a prone set-up is less comfortable than a supine one, and not all patients could maintain this treatment position. In our institution, prone set-up is proposed only to patients with large and/or pendulous breasts whose dosimetry in the supine position 
does not satisfy OAR constraints. Therefore, only a small percentage of patients, approximately $5-10 \%$ are treated in such a way. Our experience is thusly limited. The routine use of MRI in large numbers of patients is also not affordable.

However, although numerically limited, our data seem to confirm the findings published by Mast suggesting that although BGT is much better identified on MRI, the clinical gain is small, and there is probably no need for such a resource-consuming procedure in standard clinical practice [12]. Analysis of the MRI BGT distribution allowed us to define simplified contouring rules for this set-up. We are continuing with this experience, trying to obtain larger patient numbers. Regardless, our preliminary results need to be confirmed by further research.

\section{Conclusion}

After this preliminary experience, a more extensive application of contouring suggestions is planned in our institution in order to evaluate the effectiveness of our preliminary findings and, if possible, to define a countouring atlas for prone set-up.

\section{Future perspective}

Prone whole-breast irradiation is helpful in selected cases. MRI simulation and image fusion improve the accuracy of target definition. A more extensive application of this experience is planned in our institution in order to evaluate the effectiveness of our preliminary findings.

\section{Financial \& competing interests disclosure}

The authors have no relevant affiliations or financial involvement with any organization or entity with a financial interest in or financial conflict with the subject matter or materials discussed in the manuscript. This includes employment, consultancies, honoraria, stock ownership or options, expert testimony, grants or patents received or pending, or royalties.

No writing assistance was utilized in the production of this manuscript.

\section{Ethical conduct of research}

Since MRI simulation is a standard procedure for different pathologies and no contrast was needed, no specific ethics review was needed. In addition, for investigations involving human subjects, informed consent has been obtained from the participants involved.

\section{References}

Papers of special note have been highlighted as: $\bullet$ of interest; $\bullet \bullet$ of considerable interest

1. Li X, Bucholz TA, MacDonald S et al. Variability of target and normal structure delineation for breast cancer radiotherapy: an RTOG multi-institutional and multiobserver study. Int. J. Radiat. Oncol. Biol. Phys. 73(3), 944-951 (2009).

2. Vinod SK, Min M, Jameson MG et al. A review of interventions to reduce inter-observer variability in volume delianeation in radiation oncology. J. Med. Imaging Radiat. Oncol. 60(3), 393-340 (2016).

3. Offersen BV, Boersma LJ, Kirkove C et al. ESTRO consensus guideline on target volume delineation for elective radiation therapy of early stage breast cancer. Radiother. Oncol. 114, 3-10 (2015).

-• Definition of reference structures.

4. Krengli M, Masini L, Caltavuturo T et al. Prone versus supine position for adjuvant breast radiotherapy: a prospective study in patients with pendulous breasts. Radiat. Oncol. 8, 232 (2013).

5. Ramella S, Trodella L, Ippolito E et al. Whole-breast irradiation: a subgroup analysis of criteria to stratify for prone position treatment. Med. Dosim. 37, 186-191 (2012).

6. Lymberys SC, deWyngaert JK, Parhar P et al. Prospective assessment of optimal individual position (prone versus supine) for breast radiotherapy: volumetric and dosimetric correlations in 100 patients. Int. J. Radiat. Oncol. Biol. Phys. 84(4), 902-909 (2011).

- Indication of clinical target volume definition in prone set-up.

7. Veldeman 1, De Gersem W, Speleers B et al. Alternated prone and supine whole-breast irradiation using IMRT: set-up precision, respiratory movement and treatment time. Int. J. Radiat. Oncol. Biol. Phys. 82(5), 2055-2064 (2012).

8. Bartlett FR, Colgan RM, Donovan EM et al. The UK heart spare study (stage IB): randomized comparison of a voluntary breath-hold technique and prone radiotherapy after breast conserving surgery. Radiother. Oncol. 114, 66-72 (2015).

9. Kirby AM, Evans PM, Donovan EM et al. Prone versus supine positioning for whole and partial-breast radiotherapy: a comparison of non-target tissue dosimetry. Radiother. Oncol. 96, 178-184 (2010).

10. Huppert N, Jozsef G, DeWyngaert K et al. The role of a prone set up in breast radiation therapy. Front. Oncol. 1, 1-8 (2011). 
11. Buijsen J, Jager JJ, Bovendeerd J et al. Prone breast irradiation for pendulous breasts. Radiother. Oncol. 82, 337-340 (2007).

12. Mast M, Coerkamp E, Heijenbrok $M$ et al. Target volume delineation in breast conserving radiotherapy: are co-registered CT and MR images of added value? Radiat. Oncol. 9, 65 (2014).

-• Image fusion and co-registration in supine breast irradiation.

13. Giezen M, Kouwenhoven E, Scholten AN et al. Magnetic resonance imaging-versus computed tomography-based target volume delineation of glandular breast tissue (clinical target volume breast) in breast-conserving therapy: an exploratory study. Int. J. Radiat. Oncol. Biol. Phys. 81(3), 804-811 (2011).

14. Nie K, Chang D. Quantitative analysis of breast parenchymal patterns using 3D fibrogladular tissues segmented based on MRI. Med. Phys. 37(1), 217-226 (2010).

15. Dipasquale G, Wang X, Chatelain-Fontanella V et al. Automatic segmentation of breast in prone position: correlation of similarity indexes and breast pendulousness with dose/volume parameters. Radiother. Oncol. 120, 124-127 (2016). 